\title{
Mesenchymal Stem Cells Utilize CXCR4-SDF-1 Signaling for Acute, but Not Chronic, Trafficking to Gastric Mucosal Inflammation
}

\author{
Calin Stoicov $\cdot$ Hanchen Li $\cdot$ Jian Hua Liu • \\ JeanMarie Houghton
}

Received: 25 April 2013/Accepted: 25 June 2013/Published online: 20 July 2013

(C) The Author(s) 2013. This article is published with open access at Springerlink.com

\begin{abstract}
Background Helicobacter infection is the main risk factor in developing gastric cancer. Mesenchymal stem cells (MSCs) are non-hematopoietic stromal cells, which are able to differentiate into different cell lineages. MSC contribute to cancer development by forming the tumor directly, contributing to the microenvironment, or by promoting angiogenesis and metastasis. CXCR4/SDF-1 axis is used by MSC in trafficking, homing, and engraftment at chronic inflammation sites, and plays an important role in tumorigenesis.

Aim To determine if CXCR4 receptor has a role in MSC contribution to the development of Helicobacter-mediated gastric cancer.

Methods SDF-1 and CXCR4 expression in mouse gastric mucosa in the setting of acute and chronic inflammation was measured using RT-PCR. Mouse culture-adapted MSC
\end{abstract}

C. Stoicov · H. Li · J. H. Liu · J. Houghton ( $\)$

Division of Gastroenterology, Department of Medicine,

University of Massachusetts Medical School, 364 Plantation

Street, Worcester, MA 01635, USA

e-mail: jeanmarie.houghton@umassmed.edu

C. Stoicoy

e-mail: calin.stoicov@umassmed.edu

H. Li

e-mail: hanchen.li@umassmed.edu

J. H. Liu

e-mail: jian.liu@umassmed.edu

C. Stoicov · H. Li · J. H. Liu · J. Houghton

Department of Cancer Biology, University of Massachusetts

Medical School, 364 Plantation Street, Worcester,

MA 01635, USA express CXCR4. Wild-type C57BL/6 mice infected with Helicobacter felis for 6 months or controls were given IV injections of CXCR4 knock-down MSC. Animals were followed for another 4 months. Homing of MSC in the stomach was quantified using RT-PCR. MSC differentiation into gastric epithelia lineages was analyzed using immunohistochemistry and fluorescent in situ hybridization.

Results CXCR4 and SDF-1 are both upregulated in the settings of Helicobacter-induced chronic gastric inflammation. CXCR4 is fully required for homing of MSC to the stomach in acute gastric inflammation, but only partially in Helicobacter-induced gastric cancer. MSC lead to gastric intraepithelial neoplasia as early as 10 months of Helicobacter infection.

Conclusions Our results show that MSC have a tumorigenic effect by promoting an accelerated form of gastric cancer in mice. The engraftment of MSC in chronic inflammation is only partially CXCR4-dependent.

Keywords Gastric cancer - Helicobacter - Mesenchymal stem cells $\cdot$ CXCR4 $\cdot$ SDF-1

\section{Introduction}

Gastric cancer is the second most common cause of cancerrelated death, and the 4th most common form of cancer in the world. Over 900,000 new cases of gastric cancer are diagnosed annually and approximately 700,000 patients die from it each year worldwide [1, 2]. Helicobacter pylori, a class I carcinogen, is the most common risk factor of gastric cancer and one of the most common infections in the world [2]. All Helicobacter-infected patients develop gastric inflammation with or without clinical symptoms, 
but fortunately less than $1 \%$ of patients will go on to develop gastric cancer. Two-thirds of gastric cancer patients have locally advanced or metastatic disease when diagnosed, making the currently available treatments ineffective [3].

Helicobacter felis-induced chronic inflammation is required for a major contribution to the development of gastric cancer [2, 4]. The immune response of the host, in combination with host genetics, environmental factors, diet, and concomitant infections with other pathogens promote Helicobacter-induced gastric cancer [5].

Chronic inflammation has been long recognized as a risk factor in cancer, as many types of malignancies are initiated in the settings of repetitive inflammatory injuries $[6,7]$.

There are two proposed models in the development of cancer: stochastic and hierarchical models. The stochastic model states that cancer is a clonal expansion of tumorigenic cells that have accumulated mutations. The hierarchical model proposes that cancer evolves from a single tumor-initiating (stem) cell. The concept of a tumor-initiating cell (TIC) is novel and is based on the fact that not all cancer cells have an equal oncogenic potential ex vivo in animal models. The TIC is reminiscent of a stem cell based on the fact that it has self-renewal and transdifferentiation capabilities into a non-TIC [8]. The environment surrounding the TIC and subsequently the cancer cells is of paramount importance. The tumor microenvironment is made up of endothelial cells, carcinoma-associated fibroblasts (CAF), adipocytes, mesenchymal stem cells (MSCs) (including bone marrow-derived), and tumor-associated macrophages. Bone marrow-derived mesenchymal stem cells (BMD-MSC) can differentiate in vivo and in vitro into other cellular components of the tumor-supporting cells [9-11].

Bone marrow-derived cells (mesenchymal and hematopoietic stem cells) rely on the CXCR4/SDF-1 axis for trafficking, homing, and engraftment. The stromal-derived factor-1 (SDF-1, also known as CXCL12) is a 68-amino acid chemokine with a broad range of functions including lymphocyte and mononuclear cells trafficking to inflammatory sites, embryonic development, bone marrow stem cell homeostasis, and angiogenesis [12, 13].

CXCR4, is a 352-amino acid seven-span transmembrane G-protein coupled receptor and one of the receptors that binds SDF-1. Upon SDF-1 binding to CXCR4 receptor, the $\mathrm{G} \alpha$ unit of the G-protein will exchange the GDP molecule with GTP, resulting in the activation of phospholipase $\mathrm{C}$ (PLC), protein kinase C (PKC), and activation of phosphatidylinositol 3-kinase (PI3K). G $\alpha$ will simultaneously inhibit the adenylate cyclase function, therefore resulting in a reduction of cyclic AMP (cAMP) levels [14]. These events will lead to cell migration, proliferation, and cytoskeletal/actin modifications. CXCR7 is a second possible receptor for SDF-1; however, it appears that it requires CXCR4 cooperation for SDF1 binding [15]. It has been shown that CXCR7 promotes transedothelial migration, adhesion, and survival of renal progenitor cells [16], functions that are similar to those of CXCR4.

Secreted trefoil factor 2 (TFF2) is a known marker of spasmolytic peptide positive gastric cell lineages and is expressed in gastric mucous neck cells. It was recently demonstrated that TFF2 activated CXCR4, thus inducing immunologic and tumorigenic responses in vivo [17].

The CXCR4/SDF-1 axis plays an important role in normal physiology and in cancer biology. The CXCR4 receptor was shown to be over-expressed in multiple types of primary cancer [18], including gastric cancer [19] and its expression is correlated with the development of peritoneal carcinomatosis. It is theorized that peritoneal mesothelial cells over-secrete SDF-1 and may attract metastatic gastric cancer cells from a primary tumor [20]. The CXCR4/SDF-1 signaling axis is also known to promote angiogenesis, local and distal metastasis, and cell survival [21]. Both CXCR4 and SDF-1 have been shown to be upregulated in the setting of Helicobacter-induced inflammation and gastric cancer $[22,23]$. Expression of CXCR4 and SDF-1 is higher in gastric cancer with lymph node metastasis when compared to non-metastatic gastric cancer and normal gastric tissue; gastric cancer cell lines express CXCR4 and have a potent proliferation and migratory response to SDF-1 stimulation in vitro [24].

In the mouse model of Helicobacter infection, BMDC are the source of metaplasia and intraepithelial neoplasia in both the H. felis [22] and H. pylori [25] models, supporting a role for BM-MSC as the TIC for gastric cancer. Presumably, recruited cells undergo mutations due to chronic inflammation of the stomach, thus initiating cancer. Also, we have previously shown that BMD-MSC can undergo mutations at an accelerated rate in culture and, when infused into an "at risk" mouse, greatly accelerates cancer progression [26]. Combined, these findings suggest that MSC contribute to cancer by both direct and indirect mechanisms, which may depend upon when and where the oncogenic mutations occur.

We hypothesized that the CXCR4 receptor might play a key role in MSC contribution to the development of gastric cancer by recruiting and/or retaining cells in the inflamed mucosa. Therefore, using a combination of approaches, we evaluated whether CXCR4 is required for MSC trafficking to the Helicobacter-induced gastric inflammation, and whether this process promotes a more advanced form of gastric cancer. 


\section{Materials and Methods}

\section{Animals}

The University of Massachusetts Institutional Animal Care and Use Committee approved all animal work. Six- to eight-week-old male C57BL/6 mice which were viral antibody ( $\mathrm{Ab}$ )-free, parasite-free, and bacterial pathogenfree, inclusive of Helicobacter species, were purchased from Jackson Laboratories (Bar Harbor, ME, USA) and housed in microisolator cages, fed standard chow, and allowed free access to water.

\section{Helicobacter Infection}

Helicobacter felis (strain 49179 from the American Type Culture Collection, Manassas, VA, USA) was grown on Trypticase soy agar with $5 \%$ sheep blood under microaerophilic conditions at $37^{\circ}$ for 4 days. Bacteria were harvested and $1 \times 10^{7} \mathrm{CFU}$ (colony-forming units) were resuspended in $500 \mu \mathrm{l}$ total volume of Trypticase soy. Mice were restrained by hand and gavaged with $H$. felis via a 20-gauge feeding tube (Popper and Sons, New Hyde Park, NY, USA) three times at 2-day intervals.

\section{Alcohol-induced Acute Gastric Injury}

Mice were gavaged with a dose of $200 \mu \mathrm{l}$ of $50 \%$ ethanol ( $5 \mathrm{~g}$ of alcohol/kg of mouse weight) in normal saline $(\mathrm{NaCl}$ $0.9 \%, \mathrm{pH} 7.2$ ) [27]. Each mouse was given one dose a day for four successive days, then sacrificed at day 5. Each mouse weighed $30 \mathrm{~g}$.

Culture and Characterization of Culture Adapted Mesenchymal Stem Cells (MSCs)

Total marrow harvesting and MSC characterization was done as previously described $[22,26]$.

\section{Mesenchymal Stem Cells Injection in Mice}

One million RFP-labeled MSC (shScramble or shCXCR4) were strained through a $40-\mu \mathrm{m}$ strainer and suspended in $500 \mu \mathrm{l}$ of PBS. Cells were intravenously injected via tail vein $6 \mathrm{~h}$ prior to the $3 \mathrm{rd}$ dose of ethanol. Animals were euthanized $12 \mathrm{~h}$ after cell injection. In the H. felis-infected model, a similar injection was given at 6 months of infection. Mice were sacrificed 4 months after injection.

\section{Histology}

Mice were euthanized, the stomach removed, opened longitudinally along the greater curvature, and gently washed with PBS (phosphate-buffered saline). Strips of gastric tissue along the lesser curvature from the squamocolumnar junction through the pylorus were taken, fixed in $10 \%$ neutral buffered formalin for $4 \mathrm{~h}$, processed by standard methods, embedded in paraffin, cut into $5-\mu \mathrm{m}$ sections, stained with $\mathrm{H} \& \mathrm{E}$, and examined for inflammation and architectural distortion as previously described.

Sections were scored in a blinded fashion as follows: Inflammation 0: Normal; 1: Small multifocal leukocyte accumulations in mucosa; 2: Coalescing mucosal inflammation; early submucosal extension; 3 : Coalescing mucosal inflammation with prominent multifocal submucosal extension \pm follicle formation; 4: Severe diffuse inflammation of mucosa, submucosa, with or without deeper layers. Hyperplasia 0 : Normal; 1: one and one-half times normal thickness; 2: two times normal thickness with mitotic figures one-third way up to surface; 3 : three times normal thickness with mitotic figures half way up to surface; 4: four times normal thickness or greater with mitotic figures greater than half way up to the surface. Parietal cell loss, mucous cell hypertrophy, metaplasia and dysplasia; 0: no substantial alterations; $1:<5 \%$ alteration; $2: 25-50 \%$ alteration; 3: 50-75\% alteration; $4:>75 \%$ alteration [4].

\section{Quantitative PCR and RT-PCR}

A $2 \mathrm{~mm} \times 2 \mathrm{~mm}$ piece of gastric mucosa taken at the fundus/ antral border was snap-frozen at the time of necropsy. All samples were processed using standard protocols as follows: total mRNA (Trizol; Invitrogen, Grand Island, NY, USA); genomic DNA (Purelink genomic DNA mini kit; Invitrogen); reverse transcription (Omniscript RT kit; Qiagen, Valencia, CA, USA); real-time quantitative PCR (Quantitect SYBR green PCR kit; Qiagen) on a CFX96 Touch Real-Time PCR detection system (Biorad, Hercules, CA, USA).

Primers: mouse SDF-1 forward: 5'GAG AGC CAC ATC GCC AGA GC and reverse: 5'GGA TCC ACT TTA ATT TCG GGT CAA. Mouse CXCR4 forward: 5'GAC CGC CTT TAC CCC GAT AGC and reverse: 5'ACC CCC AAA AGG ATG AAG GAG TC. Mouse beta actin forward: 5'CCT AAG GCC AAC CGT GAA AAG ATG and reverse: 5'GTC CCG GCC AGC CAG GTC CAG. RFP forward: 5'CCC GCC GAC ATC CCC GAC TAC AT and reverse: 5'CTT CAG CTT CAG GGC GTG GGA GAT.

All annealing $T=59{ }^{\circ} \mathrm{C}$.

Q-PCR experiments were run 3 times with similar results.

CXCR4 Knock-Down in Mesenchymal Stem Cells

shCXCR4-GFP constructs (clones 1, 2, 3) was purchased from Genecopoeia (Rockville, MD, USA) and cells were infected with clones 1, 2 and 3 in succession. The infection efficiency was checked using GFP fluorescence. 
cAMP (Cyclic Adenosine Monophosphate) ELISA

Assay

Plates precoated with anti-cAMP antibodies (Applied Biosystems, Bedford, MA, USA) were seeded with 50,000 cells, grown overnight, then stimulated with SDF-1 at $100 \mathrm{ng} / \mathrm{ml}$ concentration for $10 \mathrm{~min}$ at $37^{\circ} \mathrm{C}$. Media were aspirated and cells lysed as per manufacturer's instructions. The results were read by a plate luminometer at an emission of $470 \mathrm{~nm}$ (Perkin-Elmer, Waltham, MA, USA). The results were plotted against the standards provided with the kit. The experiment was run 3 times with similar results.

\section{Transwell Cell Migration Assay}

One hundred microliters of mesenchymal cells at a concentration of 10,000 -cells $/ 100 \mu \mathrm{l}$ in $0.1 \%$ fetal calf serum were seeded in the well insert of Transwell plates with an $8-\mu \mathrm{m}$ pore size inserts (Corning, Lowell, MA, USA). Cells were stimulated with or without $100 \mathrm{ng} / \mathrm{ml}$ of SDF-1 overnight. Cells that migrated through the Transwell were fixed with methanol/acetic acid (3:1 ratio), stained with Giemsa solution (1:20 concentration) for $10 \mathrm{~min}$, enumerated, and plotted as a ratio of its nonstimulated control group. The experiment was run 3 times with similar results.

\section{Western Blotting}

Performed using anti-CXCR4 antibody (1/250; Abcam, UK) and secondary antibody $\beta$-actin HRP conjugated $(1 / 1,000$; Santa Cruz, CA, USA).

Immunohistochemistry (IHC) and Fluorescent In Situ Hybridization (FISH)

RFP IHC using a rabbit polyclonal antibody (1/50; Rockland, Gilbertsville, PA, USA) [26]. FISH for Y chromosome on gastric tissue sections was performed as previously described [26]. For cytokeratin detection, tissue was then exposed to anti-pankeratin PE conjugated antibody (clone C11; Abcam, Cambridge, MA, USA) at a concentration of $1 / 50$ for $20 \mathrm{~min}$ at $37^{\circ} \mathrm{C}$. Slides were mounted with DAPI antifade then immediately viewed with a fluorescent microscope (Olympus, Japan).

\section{Statistical Analysis}

Folds of relative gene expression for RFP, SDF-1, and CXCR4 are reported as a mean $\pm 1 \mathrm{SD}$ using actin as an internal control. Calculation of fold changes of real-time RT-PCR was done using the $2^{-\Delta \Delta \mathrm{Ct}}$ method; the statistical analysis was done with the REST 2009 software (Technical University, Munich, Germany). The remaining of statistical analysis was done using Prism GraphPad program (La Jolla, CA, USA). Pathology data between control group and 2,6 , and 12 months of $H$. felis infection was compared using multiple unpaired $t$ tests of two-way ANOVA. We applied the Bonferoni correction method where multiple comparisons were made. Unpaired Student's $t$ test was used to compare cAMP concentration in stimulated and unstimulated cells; data are reported as a mean \pm 1 SD. A value of $p<0.05$ was considered statistically significant.

\section{Results}

The Role of the CXCR4/SDF-1 Axis in H. felis-induced Gastric Injury

First, we established the baseline of mucosal changes to $H$. felis infection in our colony of mice. The normal gastric mucosa of mice contained: chief cells, located in the lower $1 / 3$ of the fundic mucosa; parietal cells, large eosin-positive cells inserted between the chief cells that populate the lower $2 / 3$ of the mucosa; and mucous cells, which are located in the pit section of the gastric gland. Rare inflammatory cells were seen in the submucosal layer (Fig. 1a).

At 2 months of $H$. felis infection, inflammation was evident in the submucosa (Fig. 1b, arrow), along with loss of parietal and chief cells and antralization of the glands. At 6 months of infection, there was a complete loss of parietal cells and the appearance of mucous metaplasia (Fig. 1c, arrow). At 12 months of infection, small intramucosal cystic glandular structures were formed (Fig. 1d, arrow). However, these structures did not penetrate the muscularis mucosa until 16-18 months of infection. Of the infected mice, $75 \%$ developed large antral masses, complicated by gastric outlet obstruction at 18 months of infection [4]. The histology scores at different time points after $H$. felis infection are represented in Fig. 1e.

Both SDF-1 and CXCR4 are elevated in acute inflammation environments and cancer. In order to test whether there is a role of the CXCR4/SDF-1 axis in H. felis-induced gastric inflammation, we evaluated the expression of SDF-1 and CXCR4 in the gastric mucosa of infected and control mice using RT-PCR. At 2 months of infection, there was a 30-fold increase in expression of SDF-1, and the level remained substantially elevated at 12 months (Fig. 1f). Over the same time course, there was a progressive upregulation of CXCR4 in the gastric mucosa, when compared to the control group (Fig. 1g).

CXCR4 "Loss of Function" Model in Culture-Adapted MSC

Mesenchymal stem cells constitutively express intra- and extracellular CXCR4 [28] and thus are able to respond to 


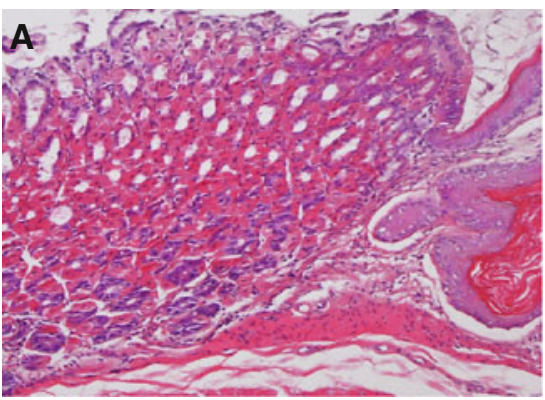

control

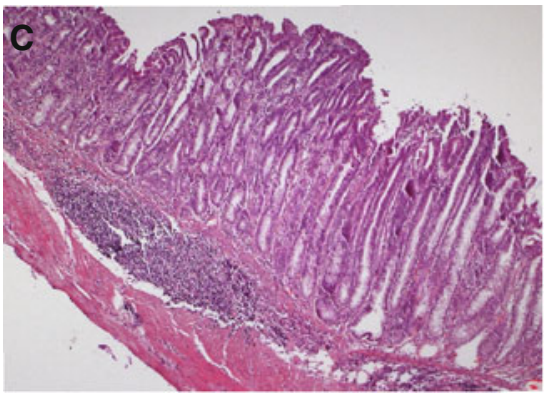

H. felis, 6 months

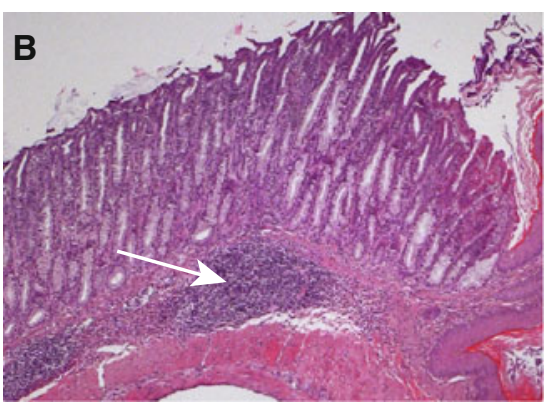

H. felis, 2 months

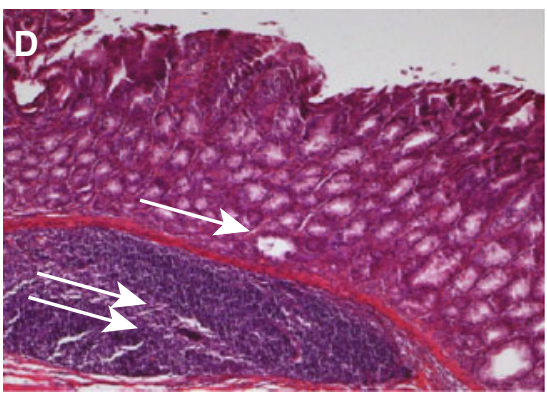

H. felis, 12 months

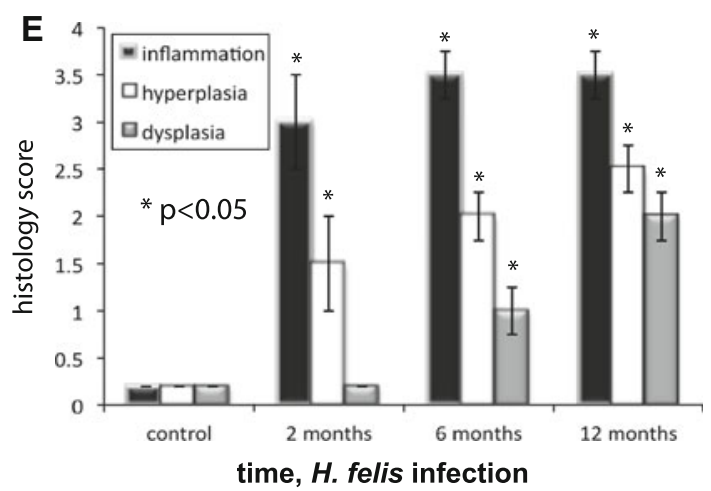

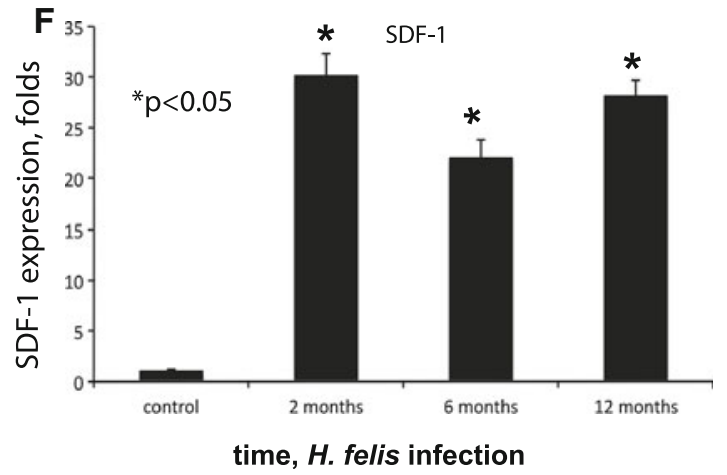

Fig. 1 Helicobacter felis infection is associated with chronic inflammation and upregulation of SDF-1 and CXCR4. Fundic mucosa taken from the lesser curvature at the squamocolumnar junction, stained with hematoxylin/eosin, magnification $\times 100$. a Control noninfected mouse; b 2 months of infection (arrow, moderate chronic submucosal inflammatory infiltrates); c 6 months of infection, mucous cell metaplasia and complete loss of parietal cells; d 12 months of

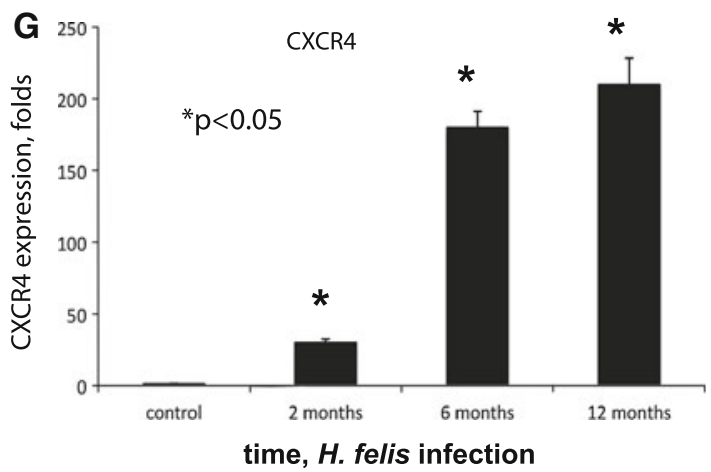

infection (single arrow points to dysplastic cystic noninvasive gastric gland in the fundus; double arrows point to massive submucosal inflammation); e histology scores from mice infected for 2,6 , and 12 months; f Gastric mucosa SDF-1 expression by RT-PCR at 2, 6, and 12 months of infection; $\mathbf{g}$ gastric mucosa CXCR4 expression at 2, 6 , and 12 months of infection $(* p<0.05)$. Error bars mean $\pm 1 \mathrm{SD}$ of the mean ( $n=4$ mice in each group) 
SDF-1 stimulation. In order to study whether CXCR4 plays a role in MSC trafficking in $\mathrm{H}$. felis-induced gastric cancer, we took a "gene knock-down" approach. RFP expressing MSC were infected in succession with three shCXCR4GFP constructs and the transfection efficiency of shCXCR4 and control construct (shScramble) was confirmed by GFP expression (Fig. 2c, f). RFP expression was preserved and did not change after shCXCR4 or shScramble transfection (Fig. 2b, e). We confirmed the CXCR4 expression levels with a western blot (Fig. $2 \mathrm{~g}$ ). The most effective inhibition of CXCR4 expression was detected in the cell line transfected with all three shCXCR4 constructs (Fig. 2g, lane 4). The growth properties (doubling time) and the appearance of shCXCR4 and shScramble cells did not change when compared to the parental cell line (data not shown).

The function of CXCR4 in both shCXCR4 and shScramble cells was tested using a cAMP concentration assay and by determining cell migration capabilities in vitro in response to SDF-1 stimulation. When stimulated by SDF-1, CXCR4 signaling occurs by dissociation of the $\mathrm{G} \beta \gamma$ subunit from the GTP bound $\mathrm{G} \alpha$. G $\alpha$ will inhibit the adenylate cyclase function, resulting in a reduction of cyclic AMP (cAMP) levels. shScramble cells, upon SDF-1 stimulation, decreased cAMP production $(p<0.05)$ confirming intact signaling, while the CXCR4-deficient cells (shCXCR4) failed to decrease the level of cAMP production $(p>0.05)$ (Fig. 2h), confirming the loss of CXCR4 expression and function.

In vitro migration was studied using a Transwell system where shCXCR4 and shScramble cells were cultured in the upper compartment of the insert of a plate separated from the lower compartment by a porous $8-\mu \mathrm{m}$ membrane. ShScramble cells responded to SDF-1 stimulation by almost tripling the number of cells that passed across the membrane and attached to the plate, when compared to the unstimulated group (Fig. 2i). On the other hand, CXCR4deficient cells did not respond to a SDF-1 gradient (Fig. 2j, cells shown as attached to the bottom of the plate; Fig. $2 \mathrm{k}$, Transwell system diagram).

Acute Alcohol Injury as a Model of Acute Gastric Inflammation in Mice

We used an acute inflammation model to test the ability of the CXCR4-deficient cells and their controls to migrate to the stomach. Acute ingestion of ethanol causes acute gastritis in mice [27]. Five gm/kg of alcohol was given daily to mice on four consecutive days; the mice were euthanized on day 5. This resulted in a mucosa, which was hemorrhagic on gross examination and showed diffuse mucosal ulceration and severe lymphocytic infiltration on microscopic examination (Fig. 3c, control; Fig. 3d, arrow). Peak inflammation occurred after the third dose of alcohol (72 h)
Fig. 2 Functional knockdown of CXCR4 prevents migration of MSC to SDF-1 in vitro. RFP-MSC transfected with GFP expressing shScramble (a-c) and GFP-shCXCR4 constructs (d-f). Magnification $\times 200$. g Western blot for CXCR4 and actin expression. $\mathbf{h}$ cAMP concentration after a 10-min SDF-1 stimulation (dark bars) and control (white bars). i Percentage of cells that migrate across the Transwell barrier to a SDF-1 stimulation, shown as the percentage of their nonstimulated group (1-100\%). $\mathbf{j}$ Giemsa staining of cell migrated to SDF-1 gradient. k Cartoon depicting of experimental design $(* p<0.05)$. Error bars mean $\pm 1 \mathrm{SD}$ of the mean

(Fig. 3b). SDF-1 was upregulated in the stomach by sixfold after 2 doses and eightfold after 3 doses of ethanol compared to the control group using RT-PCR (Fig. 3a). SDF-1 was assessed in other organs, including the colon, lung, and spleen. The stomach was the site of maximal SDF-1 upregulation (data not shown) in response to local inflammatory stimuli.

CXCR4 Is Required for MSC Trafficking in Alcoholinduced Acute Gastritis Model

We hypothesize that MSC, which possess a CXCR4 receptor, will mobilize to inflammatory sites, where SDF-1 expression is abundant. In order to test this hypothesis, we used the alcohol-induced acute gastritis model. C57BL/6 mice were given ethanol or water (control). We delivered $1 \times 10^{6}$ of either shScramble or shCXCR4 cells intravenously $6 \mathrm{~h}$ prior to the 3rd dose of ethanol, when SDF-1 were at peak levels (Fig. 3a). Animals were euthanized $12 \mathrm{~h}$ after the cells were injected. We used PCR with RFPspecific primers for the detection of injected MSC within the gastric mucosa. Under baseline conditions, CXCR4expressing cells (shScramble) were found in the stomach at 1.9-fold of control expression. Under the alcohol-induced gastritis conditions, the recovery of the shScramble was at 5.6-fold. We were unable to detect the presence of MSC in mice injected with CXCR4-deficient cells (shCXCR4) (Fig. 4a).

CXCR4 Is Partially Required for Homing of MSC to the Stomach in H. felis-induced Gastric Cancer

Helicobacter felis-induced chronic gastric inflammation results in upregulation of SDF-1, creating a permissive environment for the homing of MSC potentially through a CXCR4 mechanism. To test whether the CXCR4 receptor is required in the homing of MSC to the stomach in the setting of $H$. felis-induced chronic gastric injury, C57BL/6 mice were infected with $H$. felis for 6 months. At 6 months of infection, infected and noninfected mice were injected with $1 \times 10^{6}$ shCXCR4 or shScramble MSC via tail vein. Animals of all groups were followed for another 4 months 

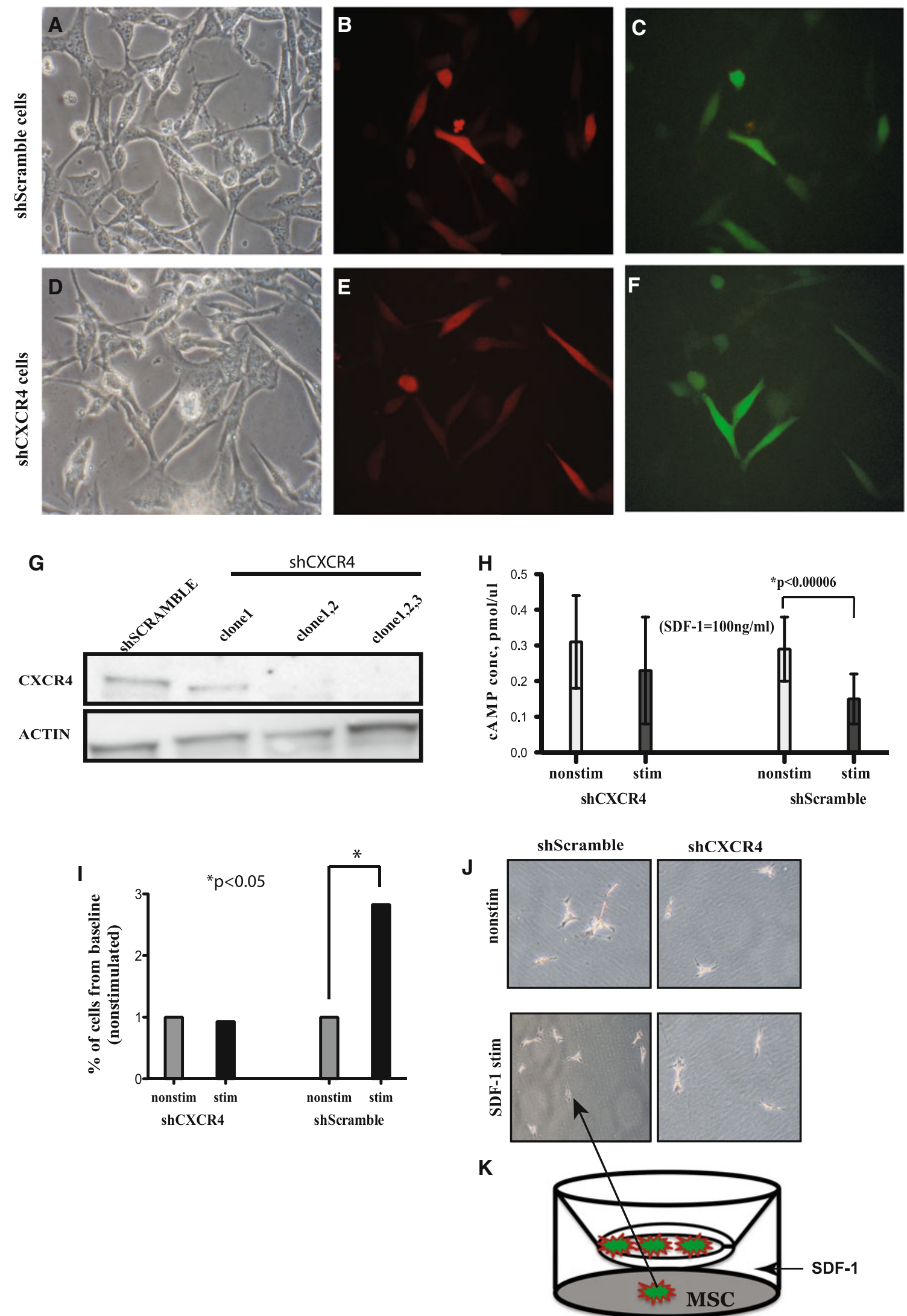

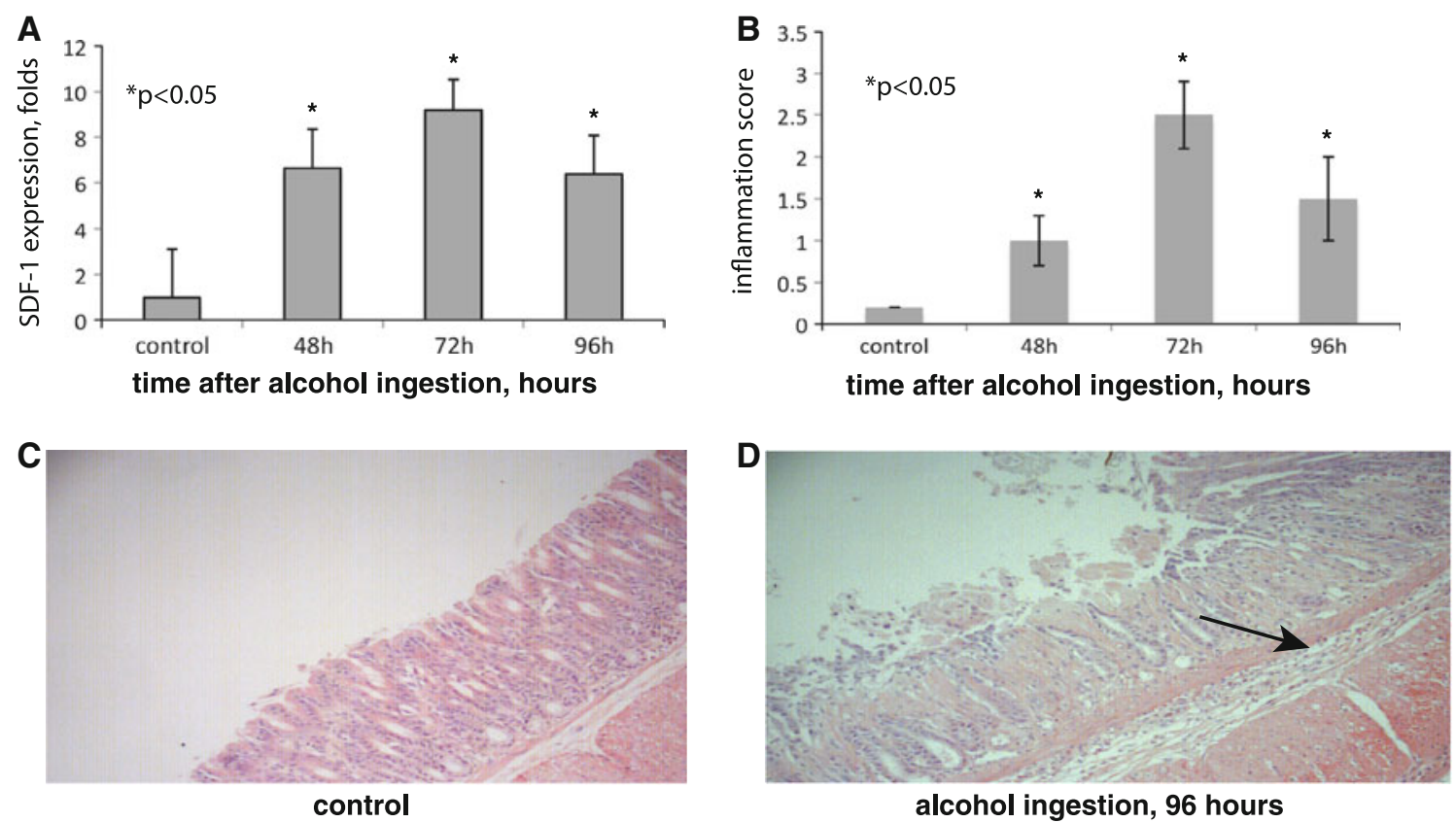

Fig. 3 Acute alcohol ingestion induces inflammation, tissue damage and overexpression of local SDF-1. a SDF-1 expression in gastric mucosa in control mice and at 48, 72, and $96 \mathrm{~h}$ after alcohol ingestion. b Inflammation scores gastric mucosa at 48, 72, and $96 \mathrm{~h}$

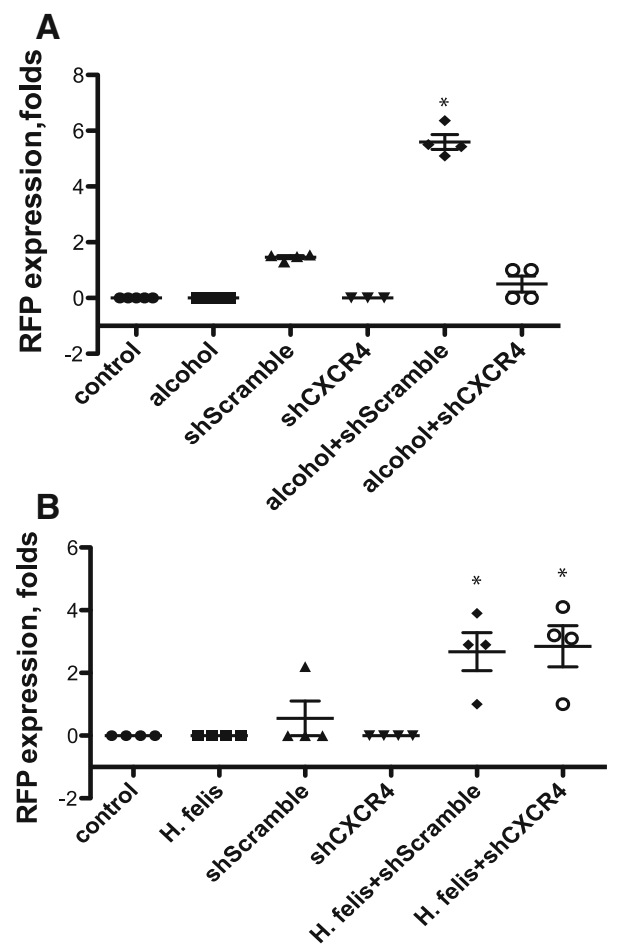

Fig. 4 MSC home to alcohol and H. felis-induced gastritis. Mice were exposed to acute ethanol ingestion or chromic $H$. felis infection and the migration of RFP-expressing MSC assessed by PCR. a PCR for RFP expression in whole gastric tissue of mice exposed to alcohol. b PCR for RFP expression in whole gastric tissue of mice exposed to H. felis infection $(* p<0.05)$. Error bars mean \pm 1 SD of the mean $(n=4$ mice in each group)

after alcohol ingestion. c, d Corresponding fundic histology at $96 \mathrm{~h}$; stained with hematoxylin/eosin, magnification $\times 100$; arrow indicates multiple inflammatory cells in the submucosa $(* p<0.05)$. Error bars mean $\pm 1 \mathrm{SD}$ of the mean ( $n=4$ mice in each group)

and then euthanized. At the time of the necropsy, all mice appeared healthy. Upon gross examination, the stomachs of the $H$. felis-infected mice that were injected with shCXCR4 and shScramble cells had an edematous and thickened fundic mucosa. In the settings of $H$. felis infection, both shScramble and shCXCR4 cells were recovered by PCR in the stomach of mice, thus demonstrating the ability of both cell types to home into the stomach. There was no RFP recovered from the control, H. felis, or noninfected shCXCR4 groups (Fig. 4c).

MSC Differentiate into Gastric Epithelial Lineages and Have a Synergistic Effect in Promoting H. felisinduced Gastric Cancer

Next, we determined the physiological effect of the homed MSC on the gastric H. felis-induced carcinogenesis. We evaluated the histology of the stomach for the presence of inflammation, hyperplasia, and intraepithelial neoplasia. $H$. felis-infected mice, with or without injected MSC, irrespective of the presence of CXCR4, had a higher stomach to total body ratio when compared with the control group (data not shown). There was no difference in the amount of inflammation seen in the stomachs of mice from the $H$. felis, $H$. felis + shScramble, or $H$. felis + shCXCR4 groups, nor was there any difference in the level of hyperplasia. Of the mice from the infected and MSCinjected groups, $75 \%$ had developed gastric intraepithelial 
Fig. 5 MSC trafficking to the gastric mucosa in the setting of chronic Helicobacter-induced inflammation results in early gastric intraepithelial neoplasia independent of CXCR4. a,

b Fundic mucosa taken from the lesser curvature at the squamocolumnar junction, stained with hematoxylin/eosin, magnification $\times 100$. a Mouse with $H$. felis infection and injection with shCXCR4 cells, Gastric Intraepithelial Neoplasia (arrow). b Mouse with $\mathrm{H}$. felis infection and injection with shScramble cells, Gastric Intraepithelial Neoplasia (arrow). c-

e Immunohistochemistry for RFP (brown staining) on a fundic mucosal strip, hematoxylin counter-staining (blue). c Control mouse, magnification $\times 100$. d Mouse with $H$. felis infection and injection with shScramble cells; magnification $\times 100$. e Mouse with $H$. felis infection and injection with shCXCR4 cells; Dysplastic gland with RFP positive staining (brown, arrow) magnification $\times 200$. $\mathbf{f}-\mathbf{h}$ FISH for Y chromosome (green staining), DAPI stained nuclei (blue) and anti-cytokeratin staining (red) of mouse stomach; magnification at $\times 200$. f Positive control, stomach of a male mouse. $\mathbf{g}$, h Mouse with $H$. felis infection and injection with shScramble cells; Y chromosome-positive cells (arrows)
A

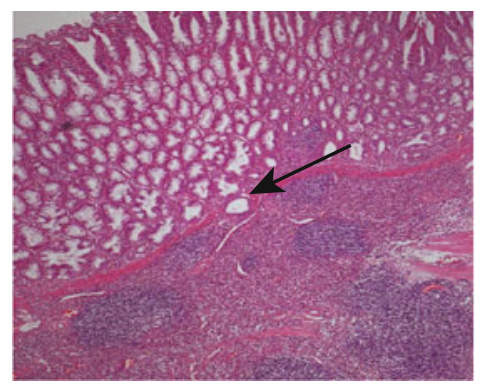

H. felis + shCXCR4

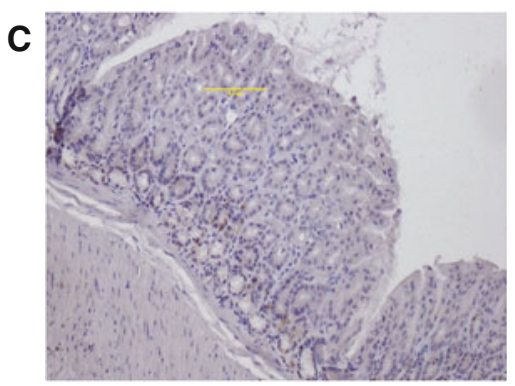

control

E

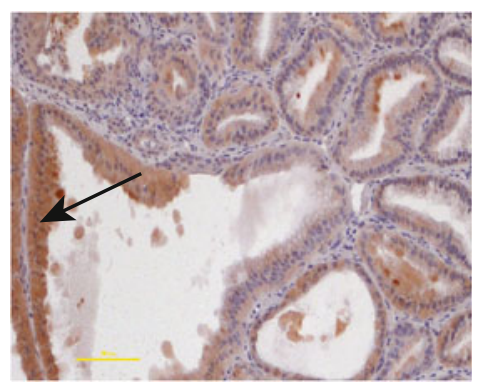

H. felis + shCXCR4

F

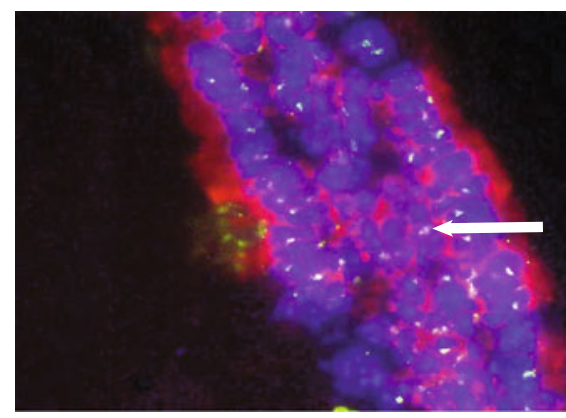

male control, stomach

H

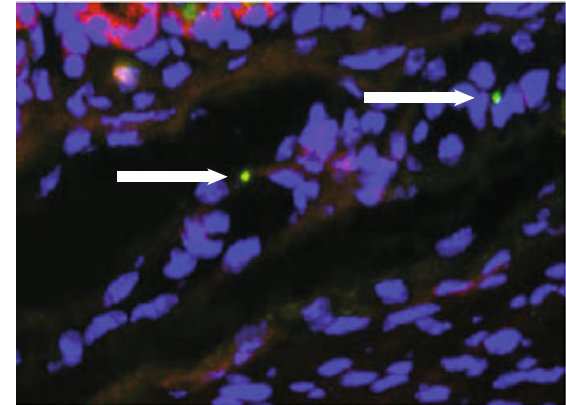

H. felis + shScramble

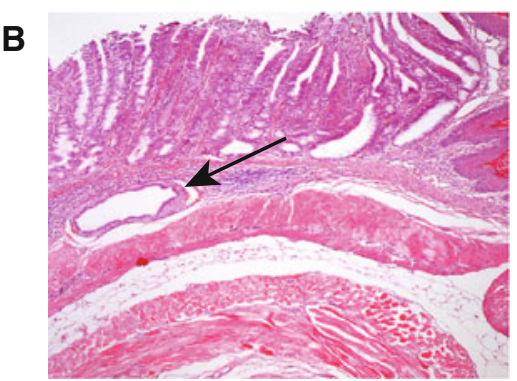

\section{H. felis + shScramble}

D

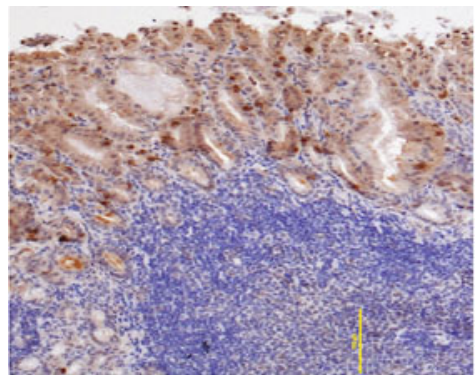

H. felis + shScramble
G

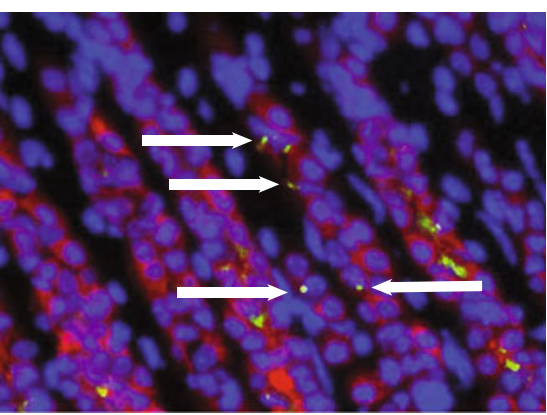

H. felis + shScramble 
neoplasia (GIN) as early as 10 months of infection. GIN represents the presence of large dysplastic glandular structures that penetrated into and beneath the muscularis mucosa of the gastric mucosa (Fig. 5a, b). This phenomenon was independent of the CXCR4 presence in the injected MSC.

The expression of RFP was assessed using an immunohistochemistry technique. RFP-positive dysplastic glands were seen throughout the stomach of $\mathrm{H}$. felisinfected and MSC-injected mice (Fig. 5d, e, brown staining). A dysplastic RFP positive gland is shown in Fig. 4e. The RFP staining was negative in the control group, which did not receive a MSC injection (Fig. 5c). FISH for Y chromosome and cytokeratin staining was used to further delineate if the MSC differentiate into gastric epithelial lineages. A gastric gland with cells containing Y chromosome staining (green, arrows), which is also positive for cytokeratin (red), is shown in Fig. 5g, arrows. In the submucosa of the same sample, there were Y-positive, but cytokeratin-negative cells of nonepithelial origin (Fig. 5h, arrows).

\section{Discussion}

Mesenchymal stem cells are non-hematopoietic stromal cells originating from bone marrow that are able to differentiate into mesenchymal (bone, cartilage, muscle) and non-mesenchymal (epithelial) cells [29]. Tumor microenvironment is a compilation of cells, which include myofibroblasts, inflammatory cells, inflammatory cells, and vascular endothelial cells; all of which are considered to be recruited from circulation or surrounding tissue with an ability to contribute to tumor development. MSC are being recruited at both primary tumor [30] and metastatic sites, mostly favoring cancer progression [31]. The contribution of MSC to the cancer development is either direct and/or indirect, through a paracrine mechanism by secreting soluble factors (cytokine, chemokines) or contribution to angiogenesis. Cancer-associated fibroblasts (CAF), which are known for their properties in forming the tumor microenvironment, were shown to originate from circulatory MSC [9]. Thus, MSC contribute directly to the tumor microenvironment or could serve as the resource of other cellular components (CAF).

Homing is the initial step in which the circulating MSC lodges within the vasculature of the injured tissue, followed by crossing of the blood-endothelium barrier using adhesion mechanisms for anchoring. The process of homing is triggered in response to injury (acute or chronic inflammation), which results in production of cytokines, chemokines, and growth factors. MSC have homing capabilities to inflamed tissues when systemically infused [32]. The interaction of SDF-1 and CXCR4 was found to play an important role in early trafficking and homing of MSC. Inflammatory cytokines, TGF- $\beta$, IL- $1 \beta$, and TNF- $\alpha$, are known for their ability to stimulate the production of matrix metalloproteinases (MMP) in MSC followed by chemotactic migration through the extracellular matrix [33]. The process of homing of MSC at the injury site is followed by engraftment, which is defined as a long-term commitment of MSC to a niche where the process of transdifferentiation into different lineages is initiated. This process goes through the replenishing effect of the peripheral stem cells. Engraftment is much more complex then homing, because it requires niche-specific signaling in order for the process of transdifferentiation to occur. In the context of chronic inflammation-derived cancer, the process of MSC niche transdifferentiation and self-renewal is exposed to oxidative stress, proinflammatory cytokines, growth factors leading to accumulation of mitotic errors and promotion of epithelial cancers through generation of cancer stem cells, and the components of tumor microenvironment, myofibroblasts, and endothelial cells.

In this study, we analyzed the mechanisms of mesenchymal stem cells trafficking into the chronic inflamed gastric mucosa in the setting of Helicobacter infection. Our initial results showed that both CXCR4 and SDF-1 are indeed upregulated in the gastric mucosa of mice infected with $H$. felis, thus demonstrating that the Helicobacterinduced gastric cancer model is indeed a favorable chronic injury environment for MSC homing and engraftment. A similar pattern of changes was revealed in human gastric cancer [20]. In order to study whether the CXCR4/SDF-1 axis plays a role in MSC trafficking to the gastric inflamed mucosa, we used a CXCR4 gene knock-down approach. First, we looked to establish an acute injury model for MSC homing in the gastric mucosa using an acute alcoholinduced gastritis model. We determined that CXCR4 was required for homing of MSC to the gastric mucosa as CXCR4-deficient cells were not detectible in the acutely injured mucosa.

Next, we tested whether CXCR4 is required for engraftment of MSC into the gastric mucosa in the settings of $\mathrm{H}$. felis-induced gastric cancer. Our results showed that, irrespective of the presence of CXCR4, MSC contributed to the development of a more advanced form of gastric cancer, as $75 \%$ of mice injected with MSC acquired GIN. This also suggested that, while acute inflammation and homing appear to be CXCR4/SDF-1-dependent, chronic inflammation and recruitment of MSC relies on alternate molecular pathways. Our findings could be explained by the fact that other chemokine receptors, for example CXCR7, might be employed by MSC to home and/or engraft into gastric mucosa in the setting of chronic $H$. felis-induced inflammation, while in the acute 
inflammation (alcohol) setting, the CXCR4/SDF-1 axis is sufficient to promote homing of MSC.

Mice receiving MSC in the setting of $H$. pylori infection had a more advanced form of gastric neoplasia, supporting the notion that MSC could be considered as a possible source of tumor initiating cells in the hierarchical model of gastric cancer. We performed immunohistochemistry (Fig. 4d, e) and FISH (Fig. 5g, h) analysis to track the MSC and their engraftment in the gastric mucosa. Injected MSC were detected in the dysplastic gastric glands (RFPpositive cells), which was further supported by the costaining for $\mathrm{Y}$ chromosome and cytokeratin. Cytokeratinnegative and chromosome $\mathrm{Y}$-positive cells detected in the submucosa may also suggest a possible contribution of MSC to the tumor-supporting cells. Understanding the molecular mechanisms of how MSC home and engraft into the chronic gastric inflammation sites, thus promoting a more advanced form of gastric cancer, is important, as it may provide clues in how to target the pathway of pathogenesis (i.e. the CXCR4/SDF-1 axis) of stomach cancer.

In conclusion, the presence of chronic inflammation recruits and retains circulating $\mathrm{MSC}$, and the retention of transformed MSC accelerates GIN. The homing of MSC acutely to the stomach is dependent on CXCR4; however, the chronic engraftment of MSC is independent of CXCR4 in the setting of Helicobacter-induced gastric cancer.

\section{Acknowledgment Grant support to J.H.: R01 CA119061.}

\section{Conflict of interest None.}

Open Access This article is distributed under the terms of the Creative Commons Attribution Noncommercial License which permits any noncommercial use, distribution, and reproduction in any medium, provided the original author(s) and the source are credited.

\section{References}

1. Parkin DM, Bray F, Ferlay J, Pisani P. Global cancer statistics, 2002. CA Cancer J Clin. 2005;55:74-108.

2. Stoicov C, Saffari R, Cai X, Hasyagar C, Houghton J. Molecular biology of gastric cancer: Helicobacter infection and gastric adenocarcinoma: bacterial and host factors responsible for altered growth signaling Gene. 2004;341:1-17.

3. Gold JS, Jaques DP, Bentrem DJ, et al. Outcome of patients with known metastatic gastric cancer undergoing resection with therapeutic intent. Ann Surg Oncol. 2007;14:365-372.

4. Cai X, Carlson J, Stoicov C, Li H, Wang TC, Houghton J. Helicobacter felis eradication restores normal architecture and inhibits gastric cancer progression in C57BL/6 mice. Gastroenterology. 2005;128:1937-1952.

5. Stoicov C, Li H, Cerny J, Houghton JM. How the study of Helicobacter infection can contribute to the understanding of carcinoma development. Clin Microbiol Infect. 2009;15:813-822.

6. IARC Working Group. Schistosomes, liver flukes and Helicobacter pylori. IARC Working Group on the Evaluation of
Carcinogenic Risks to Humans. Lyon, 7-14 June 1994 IARC Monogr Eval Carcinog Risks Hum 1994;61:1-241.

7. Hanahan D, Weinberg RA. Hallmarks of cancer: the next generation. Cell. 2011;144:646-674.

8. Visvader JE, Lindeman GJ. Cancer stem cells in solid tumours: accumulating evidence and unresolved questions. Nat Rev Cancer. 2008;8:755-768.

9. Spaeth EL, Dembinski JL, Sasser AK, et al. Mesenchymal stem cell transition to tumor-associated fibroblasts contributes to fibrovascular network expansion and tumor progression. PLoS One. 2009;4:e4992.

10. Mishra PJ, Humeniuk R, Medina DJ, et al. Carcinoma-associated fibroblast-like differentiation of human mesenchymal stem cells. Cancer Res. 2008;68:4331-4339.

11. Bergfeld SA, DeClerck YA. Bone marrow-derived mesenchymal stem cells and the tumor microenvironment. Cancer Metastasis Rev. 2010;29:249-261.

12. Bleul CC, Fuhlbrigge RC, Casasnovas JM, Aiuti A, Springer TA. A highly efficacious lymphocyte chemoattractant, stromal cellderived factor 1 (SDF-1). J Exp Med. 1996;184:1101-1109.

13. Luster AD. Chemokines-chemotactic cytokines that mediate inflammation N. Engl J Med. 1998;338:436-445.

14. Hamm HE. The many faces of G protein signaling. J Biol Chem. 1998;273:669-672.

15. Cubedo N, Cerdan E, Sapede D, Rossel M. CXCR4 and CXCR7 cooperate during tangential migration of facial motoneurons. $\mathrm{Mol}$ Cell Neurosci. 2009;40:474-484.

16. Mazzinghi B, Ronconi E, Lazzeri E, et al. Essential but differential role for CXCR4 and CXCR7 in the therapeutic homing of human renal progenitor cells. J Exp Med. 2008;205:479-490.

17. Dubeykovskaya Z, Dubeykovskiy A, Solal-Cohen J, Wang TC. Secreted trefoil factor 2 activates the CXCR4 receptor in epithelial and lymphocytic cancer cell lines. J Biol Chem. 2009;284: 3650-3662.

18. Domanska UM, Kruizinga RC, Nagengast WB, et al. A review on CXCR4/CXCL12 axis in oncology: No place to hide. Eur $J$ Cancer. 2012;49:219-230.

19. Kwak MK, Hur K, Park DJ, et al. Expression of chemokine receptors in human gastric cancer. Tumour Biol. 2005;26:65-70.

20. Yasumoto K, Koizumi K, Kawashima A, et al. Role of the CXCL12/CXCR4 axis in peritoneal carcinomatosis of gastric cancer. Cancer Res. 2006;66:2181-2187.

21. Balkwill F. Cancer and the chemokine network. Nat Rev Cancer. 2004;4:540-550.

22. Houghton J, Stoicov C, Nomura S, et al. Gastric cancer originating from bone marrow-derived cells. Science. 2004;306:1568-1571.

23. Ohmae T, Hirata Y, Maeda S, et al. Helicobacter pylori activates NF-kappaB via the alternative pathway in B lymphocytes. $J$ Immunol. 2005;175:7162-7169.

24. Zhao BC, Wang ZJ, Mao WZ, et al. CXCR4/SDF-1 axis is involved in lymph node metastasis of gastric carcinoma. World $J$ Gastroenterol. 2011;17:2389-2396.

25. Varon C, Dubus P, Mazurier F, et al. Helicobacter pylori infection recruits bone marrow-derived cells that participate in gastric preneoplasia in mice. Gastroenterology. 2012;142:281-291.

26. Li H, Fan X, Kovi RC, et al. Spontaneous expression of embryonic factors and $\mathrm{p} 53$ point mutations in aged mesenchymal stem cells: a model of age-related tumorigenesis in mice. Cancer Res. 2007;67:10889-10898.

27. Andrade MC, Menezes JS, Cassali GD, Martins-Filho OA, Cara DC, Faria AM. Alcohol-induced gastritis prevents oral tolerance induction in mice Clin. Exp Immunol. 2006;146:312-322.

28. Wynn RF, Hart CA, Corradi-Perini C, et al. A small proportion of mesenchymal stem cells strongly expresses functionally active CXCR4 receptor capable of promoting migration to bone marrow. Blood. 2004;104:2643-2645. 
29. Jiang Y, Jahagirdar BN, Reinhardt RL, et al. Pluripotency of mesenchymal stem cells derived from adult marrow. Nature. 2002;418:41-49.

30. Studeny M, Marini FC, Champlin RE, Zompetta C, Fidler IJ, Andreeff M. Bone marrow-derived mesenchymal stem cells as vehicles for interferon-beta delivery into tumors. Cancer Res. 2002;62:3603-3608.

31. Dhar K, Dhar G, Majumder M, et al. Tumor cell-derived PDGF-B potentiates mouse mesenchymal stem cells-pericytes transition and recruitment through an interaction with NRP-1. Mol Cancer. 2010;9:209.

32. Chavakis E, Urbich C, Dimmeler S. Homing and engraftment of progenitor cells: a prerequisite for cell therapy. J Mol Cell Cardiol. 2008;45:514-522.

33. Ries C, Egea V, Karow M, Kolb H, Jochum M, Neth P. MMP-2, MT1-MMP, and TIMP-2 are essential for the invasive capacity of human mesenchymal stem cells: differential regulation by inflammatory cytokines. Blood. 2007;109:4055-4063. 